\title{
EXACT SOLUTION AND BUCKLING CONFIGURATION OF NANOTUBES CONTAINING INTERNAL FLOWING FLUID
}

\author{
B.Gültekin Sınır, F.Esra Uz and Sefa Ergun \\ Celal Bayar University, Faculty of Engineering, Department of Civil Engineering \\ 45140, Muradiye, Manisa \\ gultekin.sinir@cbu.edu.tr, fatmaesrauz@ogr.cbu.edu.tr, sefa.ergun@cbu.edu.tr
}

\begin{abstract}
In this study, the post-divergence behaviour of nanotubes of conveying internal moving fluid with both inner and outer surface layers are analyzed in nonlinear theorical model. The governing equation has the cubic nonlinearity. The source of this nonlinearity is the surface effect and mid-plane stretching in the nanobeam theory. Exact solutions for the post buckling configurations of nanotubes with clamped-hinged with torsionally spring and hybrid boundary conditions is found. The critical flow velocity at which the nanotube is buckled is shown. The effects of various nondimensional system parameters on the post-buckling behaviour are investigated.
\end{abstract}

Key Words- Nanotube, Surface effect, Nonlinear, Exact solution, Post-buckling

\section{INTRODUCTION}

Nanotubes/nanobeams are of great value in nanotechnology practice. Large modulus of elasticity and small specific weight of carbon nanotubes make them materials for applications in the nanotechnology. One of the significant properties of such structures is the ability of storing and transporting of fluid-like materials (e.g., water, gases and nanoparticle). Due to these properties, nanobeams and nanopipettes have multifarious applications in nanomechanical systems and nanobiological devices [1], drug delivery devices, fluid filtration devices [2], fluid transport, fluid stroge, biosensors [3], atomicforce microscopes, nano-fibers for composite materials [4], atomic force microscope [5].

We will study on a classify of nano-fluidic devices that may be characterized as nanotubes conveying internal moving fluid, transporting fluid. Buckling problem and post-buckling analyses of the nanotubes are of great importance in engineering science. The main aim of these studies of buckling problems is to find postbuckling configurations of the nanobeams.

In the paper [6] buckling and post-buckling of long pressurized elastic thin-walled tubes under in-plane bending are presented. [7] Exact solutions for the post buckling configurations of beams and the dynamic stability of the obtained postbuckling configurations are presented. [8] Thermal buckling and post-buckling behavior is investigated for functionally graded carbon nanotube reinforced composite plates by single-walled carbon nanotubes subjected to in-plane temperature variation. In the essay [9] is to present exact and effective expressions for the postbuckling configurations of single-walled carbon nanotubes with various conditions. [10] The post-buckling 
behavior of supported nanobeams is studied. The effects of nanobeam length, bulk thickness and several dimensionless parameters on the post-buckling behavior are investigated in the paper. [11] Post-buckling bifurcations and stability of high-speed axially moving beams are analyzed. [12] Buckling and post-buckling analysis of fluid conveying multi-walled carbon nanotubes are analytically examined.

In this paper, exact solutions for the post buckling configurations of nanobeams are exhibited. We present exact solution post buckling configurations of nanobeams with clamped-hinged with torsionally spring and hybrid boundary conditions. The equilibria of the axially moving beam in the supercritical regime have given analytically [13]. When the geometric nonlinearity accounting for midplane stretching is taken into consideration, the nonlinear buckling problem can be obtained [7]. The essential aims of studies of buckling problems are to find their associated buckled shapes and critical flow velocity which is relative to the surface effect.

\section{GOVERNING EQUATION}

A nanobeam/nanotube with both ends supported for transporting fluid is considered as a hollow cylindrical tube. The internal flow is modeled as a continuum flow. The outside nanobeam, made up of a bulk part and two additional thin surface layers (inner and outer layers). The mechanical features of the bulk part are elasticity modulus $E$ and mass density $\rho_{b}$. It is assumed that the inner and outer diameters of the bulk part are $d_{i}$ and $d_{o}$. The thickness of each surface layer is taken as $t_{0}$. The inner and outer surface layers are with surface elasticity modulus of $E_{s}, \tau_{0}$ denote the surface residual tension.

In this study, the surface layers in nano-materials essential have two additional apparent effects. The first effect relates to the surface residual tension which acts as distributed transverse loads. The second effect is relative to the increasing flexural rigidity.

The linear governing equation of the nanobeam is given by [14]

$(E I+h) \frac{\partial^{4} \widehat{w}}{\partial \bar{x}^{4}}+\left(M U^{2}-\Pi_{0}\right) \frac{\partial^{2} \widehat{w}}{\partial \widehat{x}^{2}}+2 M U \frac{\partial^{2} \widehat{w}}{\partial \widehat{x} \partial \widehat{t}}+(m+M) \frac{\partial^{2} \widehat{w}}{\partial \hat{t}^{2}}=0$

where, $\widehat{w}(\widehat{x}, \widehat{t})$ denotes the transverse deflection, $\hat{x}$ and $\hat{t}$ are the axial coordinate and time, $E I$ is defined as the flexural rigidity of the empty nanobeam without surface layers, $M$ is the mass of fluid per unit length, flowing with a constant flow velocity $U$, $m$ is the mass of the empty nanobeam per unit length.

The above mentioned equation, $h$ exhibits the additional bending rigidity due to two surface layers (inner and outer layers) and the constant $\Pi_{0}$ is an axial force relative to the residual surface stress. $-\Pi_{0} \partial^{2} \widehat{w} / \partial \hat{x}^{2}$ in Eq. (1) is a distributed transverse loading. In accordance with Ref. [14], the additional bending rigidity on account of surface layers may be shown as

$h=\frac{1}{8} \pi E_{s} t_{0}\left(d_{i}^{3}+d_{o}^{3}\right)$

and the axial force $\Pi_{0}$ is written by

$\Pi_{0}=2 \tau_{0}\left(d_{i}+d_{o}\right)$

In accordance with [15], for a beam supported between axially immobile supports, the non-linearity related to the mean axial extension of the beam may be viewed as the 
dominating non-linear factor, so the problem governing the buckling of beams accounting for midplane stretching is given by

$$
\begin{aligned}
& (E I+h) \frac{\partial^{4} \widehat{w}}{\partial \widehat{x}^{4}}+\left(M U^{2}-\Pi_{0}\right) \frac{\partial^{2} \widehat{w}}{\partial \widehat{x}^{2}}+2 M U \frac{\partial^{2} \widehat{w}}{\partial \widehat{x} \partial \widehat{t}}+(m+M) \frac{\partial^{2} \widehat{w}}{\partial \widehat{t}^{2}}= \\
& \frac{E A+E_{s} t_{0} \pi\left(d_{i}+d_{o}\right)}{2 L} \frac{\partial^{2} \widehat{w}^{L}}{\partial \widehat{x}^{2}} \int_{0}\left(\frac{\partial \widehat{w}}{\partial \widehat{x}}\right)^{2} d \widehat{x}
\end{aligned}
$$

The nonlinear term added in Eq. (4) is a uniform additional tensile load. This makes owing to analyze the post-instability answers of the flow-conveying nanobeams [10].

We use the following non-dimensional variables:

$$
\begin{aligned}
& x=\frac{\widehat{x}}{L}, w=\frac{\widehat{w}}{r}, t=\widehat{t} \sqrt{\frac{E I}{(M+m) L^{4}}}, u=U \sqrt{\frac{M L^{2}}{E I}}, \beta=\frac{M}{M+m} \\
& \alpha=\frac{h}{E I} \cong \frac{\kappa}{4}, \quad \gamma=\frac{\Pi_{0} L^{2}}{E I}, \kappa=\frac{E_{s} t_{0}}{E\left(d_{o}-d_{i}\right)}
\end{aligned}
$$

where $r=\sqrt{I / A}$ is the radius of gyration of the cross-section. Where $A$ is the crosssectional area of the bulk wall.

As a result, we rewrite Eq. (4) of motion in dimensionless form as follows:

$$
\left(1+\frac{\kappa}{4}\right) \frac{\partial^{4} w}{\partial x^{4}}+\left(u^{2}-\gamma\right) \frac{\partial^{2} w}{\partial x^{2}}+2 u \sqrt{\beta} \frac{\partial^{2} w}{\partial x \partial t}+\frac{\partial^{2} w}{\partial t^{2}}=\frac{1}{2}(1+\kappa) \frac{\partial^{2} w}{\partial x^{2}} \int_{0}^{1}\left(\frac{\partial w}{\partial x}\right)^{2} d x
$$

They have significant effects on vibration and stability of the distributed parameter systems. The clamped-hinged with torsionally spring and hybrid boundary conditions could be a more suitable description than the fixed or hinged supported ends. For each set of boundary conditions, the nanobeam would buckle at a critical flow velocity. Setting transverse displacements to zero and balancing bending moment at both ends lead to the dimensionless boundary conditions:

Clamped-hinged with torsionally spring boundary conditions:

$$
w(0, t)=w(1, t)=0, \quad w^{\prime}(0, t)=0, \quad w^{\prime \prime}(1, t)=-\eta w^{\prime}(1, t)
$$

Hybrid boundary conditions:

$$
w(0, t)=w(1, t)=0, w^{\prime \prime}(0, t)=\eta_{1} w^{\prime}(0, t), \quad w^{\prime \prime}(1, t)=-\eta_{2} w^{\prime}(1, t)
$$

when $\eta \rightarrow \infty$, Eq. (7-8) yields clamped end supports. On the other hand, simply supported ends occur when $\eta \rightarrow 0$.

\section{NONLINEAR EQUILIBRIUM SOLUTIONS}

The equation of a tube containing internal flowing fluid is similar to the equation of a beam subjected to a compressive load. The centrifugal force in the equation of pipes acts in the same manner as a compressive load $[10,16]$. It has been noticed that equilibrium solutions of the pipe are analogous to those in a buckled problem [10].

The buckling problem can be obtained from Eq. (6) by dropping the time dependent, and forcing terms and denoting the buckled configuration by $\psi(x)$. The result is

$\left(1+\frac{\kappa}{4}\right) \frac{\partial^{4} \psi}{\partial x^{4}}+\left(u^{2}-\gamma\right) \frac{\partial^{2} \psi}{\partial x^{2}}=\frac{1}{2}(1+\kappa) \frac{\partial^{2} \psi}{\partial x^{2}} \int_{0}^{1}\left(\frac{\partial \psi}{\partial x}\right)^{2} d x$

where $\psi(x)$ is the buckled configuration related to the velocity $u$. 
Actually, above equation is the nonlinear integro-differential equation. We note that the integral in the equation is a constant for a given $\psi(x)$ :

$\Gamma=\int_{0}^{1}\left(\frac{\partial \psi}{\partial x}\right)^{2} d x$

where $\Gamma$ is a constant. As a result Eq. (9) becomes

$\frac{\partial^{4} \psi}{\partial x^{4}}+\lambda^{2} \frac{\partial^{2} \psi}{\partial x^{2}}=0$

where

$\lambda^{2}=\frac{u^{2}-\gamma}{1+\kappa / 4}-\frac{1+\kappa}{2(1+\kappa / 4)} \Gamma$

Above relevant gives the critical velocity on the nonlinear system. The general solution of Eq.(11) can be written as

$\psi(x)=c_{1}+c_{2} x+c_{3} \cos \lambda x+c_{4} \sin \lambda x$

To determine the arbitrary constants $c_{n}$, the boundary conditions are enforced. This application yields four algebraic equations in $c_{n}$. Applying boundary conditions, we obtain four algebraic equations. For non-trivial solution, the determinant of the coefficients matrix of these algebraic equations must be equal to zero. That is the eigenvalue problem for $\lambda$.

The eigenvalues of the coefficients matrix give the divergence fluid velocity known as the critic velocity. As a result, for a given buckling velocity, the buckled configuration, $\psi(x)$, is obtained in a closed form.

The substitution of Eq.(13) into (12) gives

$\lambda^{2}=\frac{u^{2}-\gamma}{1+\kappa / 4}-\frac{1}{4} c^{2} \lambda^{2} \frac{1+\kappa}{1+\kappa / 4}$

or the mid-span rise of buckling can be written

$c= \pm 2 \sqrt{\frac{u^{2}-\gamma}{(1+\kappa) \lambda^{2}}-\frac{1+\kappa / 4}{1+\kappa}}$

\subsection{Clamped-hinged with torsionally spring}

The supports are modeled by clamped-hinged boundary conditions:

$\psi(0)=\psi(1)=0, \psi^{\prime}(0)=0, \psi^{\prime \prime}(1)=-\eta \psi^{\prime}(1)$

The coefficient matrix is given by

$\left[\begin{array}{cccc}1 & 0 & 1 & \sin \lambda \\ 1 & 1 & \cos \lambda & 0 \\ 0 & 1 & 0 & (\eta \cos \lambda-\lambda \sin \lambda) \lambda\end{array}\right]\left\{\begin{array}{l}c_{1} \\ c_{2} \\ c_{3} \\ c_{4}\end{array} \quad \eta \quad(-\lambda \cos \lambda+\eta \sin \lambda)=\left\{\begin{array}{l}0 \\ 0 \\ 0 \\ 0\end{array}\right\}\right.$

Demanding that the determinant of the coefficient matrix equals zero, we obtain the following characteristic equation for $\lambda$ :

$(\lambda \cos \lambda-\sin \lambda) \lambda+\eta(\lambda \sin \lambda-2+2 \cos \lambda)=0$

When $\eta \rightarrow \infty$ clamped end supports. In the case of clamped-clamped nanobeams then the characteristic equation becomes 
$\lambda \sin \lambda-2+2 \cos \lambda=0$

Simply supported ends occur when $\eta \rightarrow 0$. In the case of clamped-hinged nanobeams then the characteristic equation becomes

$\lambda \cos \lambda-\sin \lambda=0$

The results are the same as Ref. [7].

From the boundary conditions, the buckling configuration $\psi(x)$ can be written as

$\psi(x)=c\left(\frac{\cos \lambda-1}{\sin \lambda-\lambda}(\lambda x-\sin \lambda x)+\cos \lambda x-1\right)$

\subsection{Hybrid boundary conditions}

The supports are modeled by hybrid boundary conditions:

$\psi(0)=\psi(1)=0, \psi^{\prime \prime}(0)=\eta_{1} \psi^{\prime \prime}(0), \psi^{\prime \prime}(1)=-\eta_{2} \psi(1)$

The coefficient matrix is given by

$\left[\begin{array}{cccc}1 & 0 & 1 & 0 \\ 1 & 1 & \cos \lambda & \sin \lambda \\ 0 & -\eta_{1} & -\lambda^{2} & -\eta_{1} \lambda \\ 0 & \eta_{2} & -\lambda^{2} \cos \lambda-\eta_{2} \lambda \sin \lambda & \eta_{2} \lambda \cos \lambda-\lambda^{2} \sin \lambda\end{array}\right]\left\{\begin{array}{l}c_{1} \\ c_{2} \\ c_{3} \\ c_{4}\end{array}\right\}=\left\{\begin{array}{l}0 \\ 0 \\ 0 \\ 0\end{array}\right\}$

The following characteristic equation for $\lambda$ :

$\lambda\left(-\lambda^{3} \sin \lambda+\lambda^{2} \eta_{2} \cos \lambda+\eta_{1} \lambda^{2} \cos \lambda+\eta_{1} \eta_{2} \lambda \sin \lambda-2 \eta_{1} \eta_{2}+2 \eta_{1} \eta_{2} \cos \lambda-\eta_{1} \lambda \sin \lambda-\eta_{2} \lambda \sin \lambda\right)=0$

When $\eta_{1,2} \rightarrow \infty$, Eq.(9) yields following relation for clamped-clamped support condition

$\lambda \sin \lambda-2+2 \cos \lambda=0$

Simply supported ends occur when $\eta_{1} \rightarrow \infty$ and $\eta_{2} \rightarrow 0$. In the case of clamped-hinged nanobeams then the characteristic equation becomes

$\lambda \cos \lambda-\sin \lambda=0$

In a similar way, below relation for hinged-hinged support is obtained by using $\eta_{1,2} \rightarrow 0$

$\sin \lambda=0$

The results are the same as Ref. [7 ].

The buckling configuration $\psi(x)$ then becomes

$\psi(x)=c\left[1-\frac{\left(\eta_{1} \lambda \cos \lambda+\eta_{1} \eta_{2} \sin \lambda-\lambda^{2} \sin \lambda+\eta_{2} \lambda \cos \lambda\right) \lambda}{\left(\lambda \sin \lambda+\eta_{2}-\eta_{2} \cos \lambda\right) \eta_{1}} x-\cos \lambda x+\frac{\eta_{1} \lambda \cos \lambda+\eta_{2} \lambda+\eta_{1} \eta_{2} \sin \lambda}{\left(\lambda \sin \lambda+\eta_{2}-\eta_{2} \cos \lambda\right) \eta_{1}} \sin \lambda x\right]$

\section{NUMERICAL RESULTS}

In Fig. 1 and Fig. 2 variation of the static deflection with the axial velocity are calculated for the three cases of clamped-clamped, clamped-hinged, and hinged-hinged supported pipes and the stability of the buckled configurations are examined. The first three buckled configurations for three different well known boundary conditions is investigated. Respectively, the ordinate and abscissa are the dimensionless transverse displacement of the pipe and the non-dimensional flow velocity. While continuous lines represent stable static regions, dotted lines represent unstable equilibrium positions. All 
the supported pipes are stable at their original static equilibrium position, up to the first critical velocity, where they unstable in a supercritical pitchfork bifurcation.

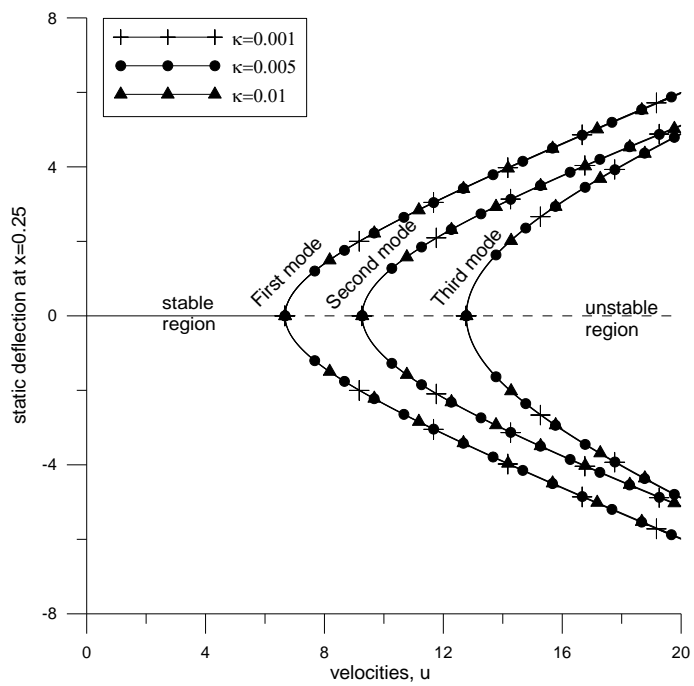

(a)

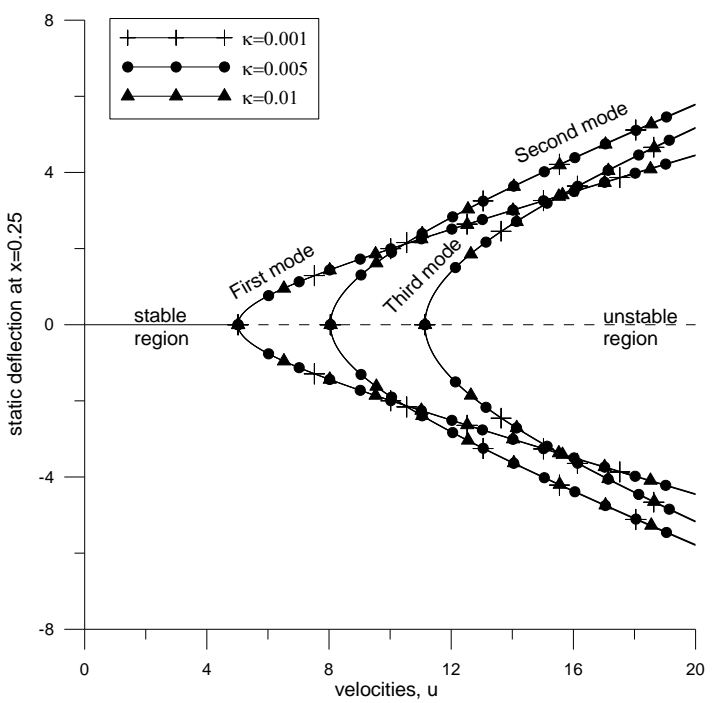

(b)

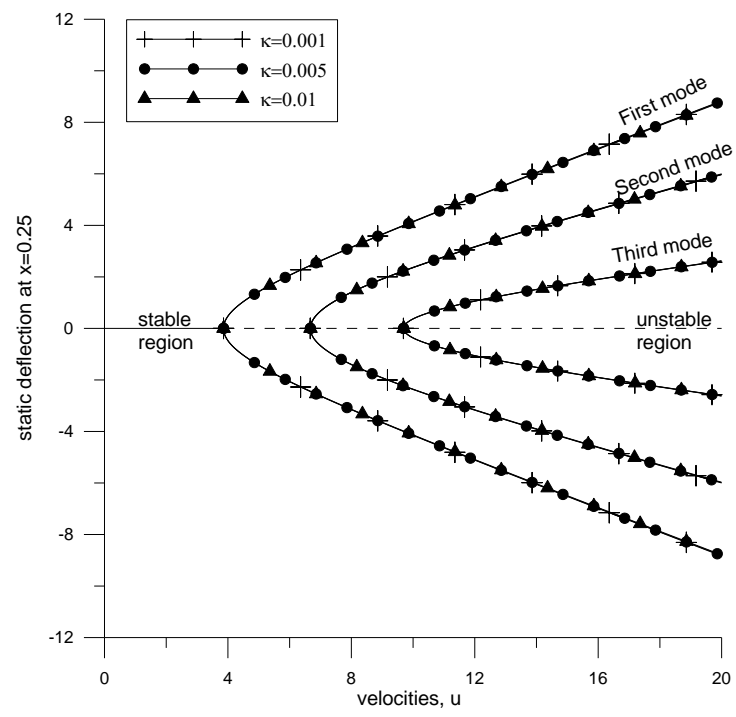

(c)

Figure 1. Bifurcation diagram of the first three mode for the $\gamma=5$, (a) clampedclamped, (b) clamped-hinged and (c) hinged-hinged supported pipe conveying fluid

In Fig. 1 as the velocity exceeds the first critical velocity, e.g., $u_{c}=6.66995$ for the clamped-clamped pipe $\kappa=0.001$ and $\gamma=5$, the straight configuration loses stability by a supercritical pitchfork bifurcation and the pipe buckles. It can be seen in Fig.1 that the effect of $\kappa$ on critical velocity can be disregarded at drawn all modes. 


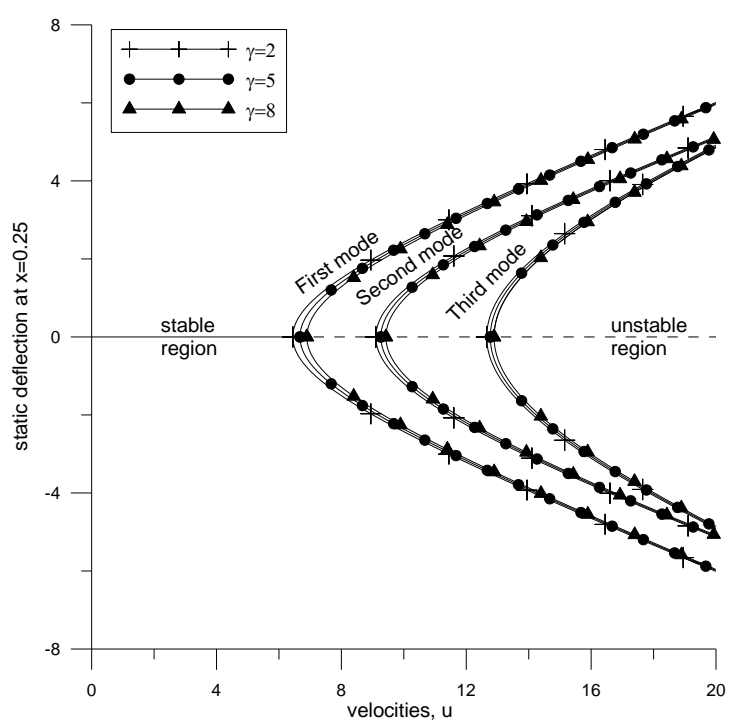

(a)

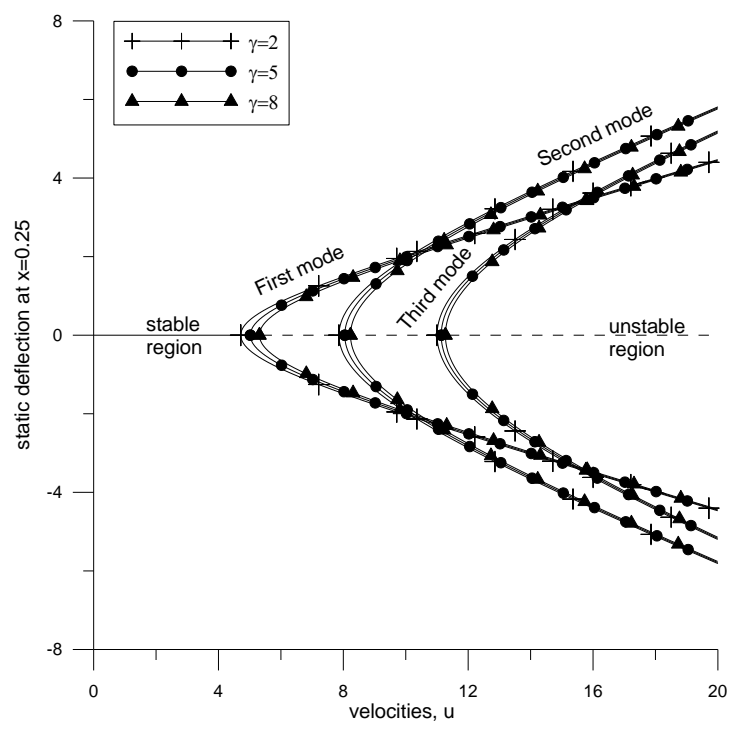

(b)

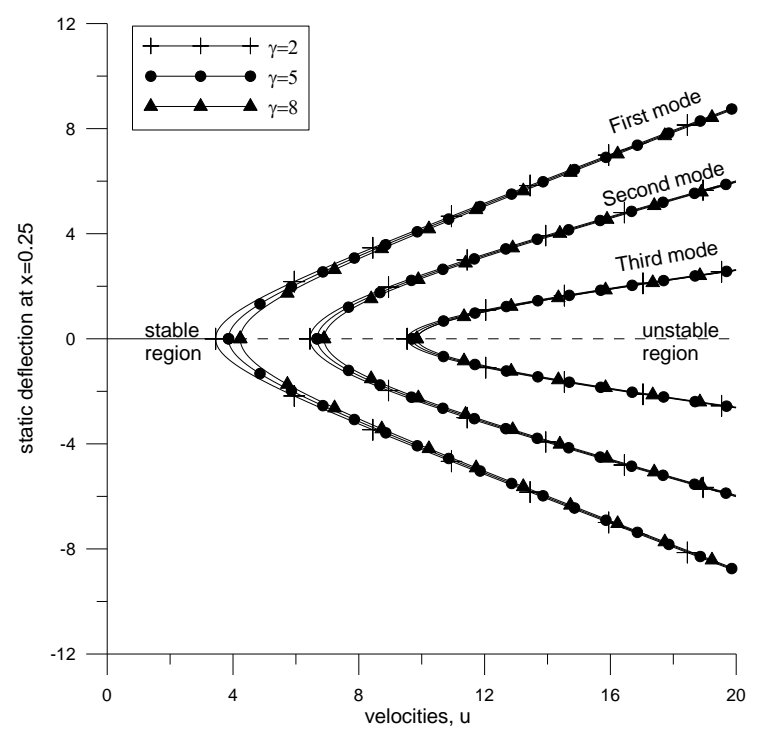

(c)

Figure 2. Bifurcation diagram of the first three mode for the $\kappa=0.005$, (a) clampedclamped, (b) clamped-hinged and (c) hinged-hinged supported pipe conveying fluid

In Fig. 2 as the velocity exceeds the first critical velocity,e.g., $u_{c}=6.4442$ for the clamped-clamped pipe $\gamma=2$ and $\kappa=0.005$, the straight configuration loses stability by a supercritical pitchfork bifurcation and the pipe buckles. The deflection amplitude of buckling increases by increasing the flow velocity beyond the critical velocity. It can be seen that in Fig.2. that $\gamma$ has meaningfull effect on critical velocities for the first three modes. 


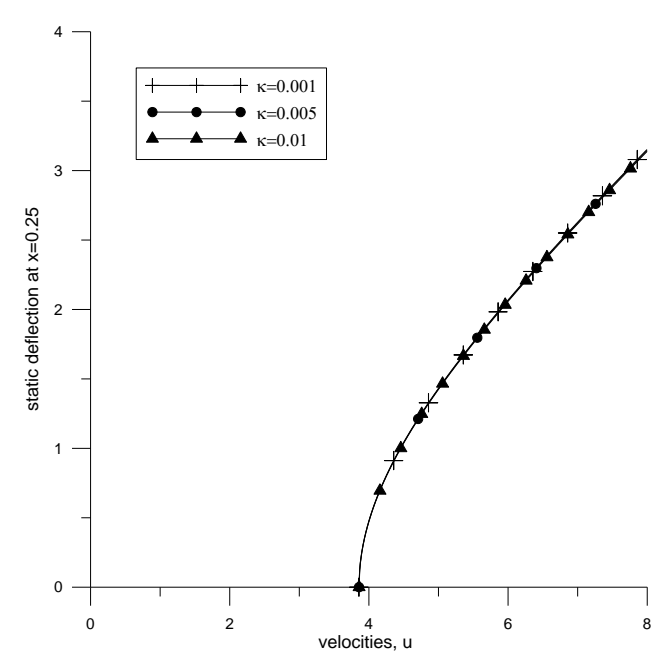

(a)

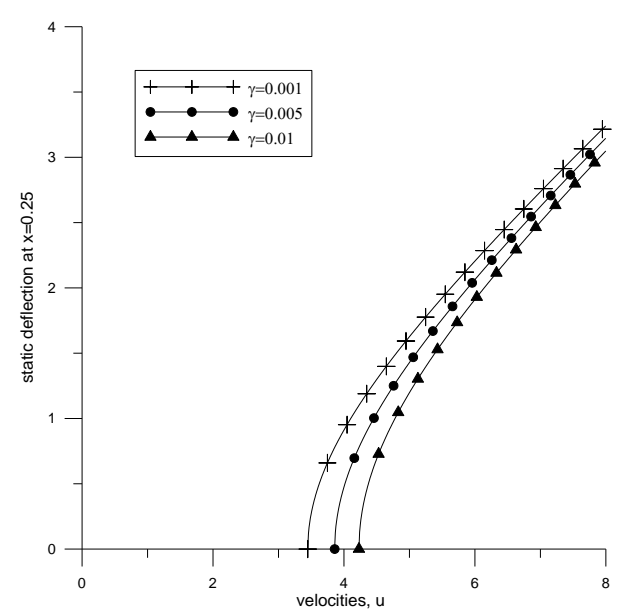

(b)

Figure 3. Bifurcation diagram for the system with different values of tension for hingedhinged supported pipe conveying fluid, (a) for the $\gamma=5$, (b) for the $\kappa=0.005$

To exhibit the influence of supplemantery stiffness term and the axial tension on the stability, Fig.3 is graphed. In Fig. 3, the influence of tension on the behavior of a hinged-hinged pipe is analyzed. Similar case is estimated for clamped-clamped and clamped-hinged boundary conditions. The amplitude of deflection of the straight pipe can be upwards or downwards, depending on initial conditions. By increasing the external tension, the critical flow velocity for divergence increases; also, at a certain flow velocity,the amplitude of buckling decreases. The amplitude of buckling can be calculated using Eq. (15). In Fig. 3 (a), the effect of $\kappa$ on the amplitude is very small, it can not even be seen. In Fig. 3 (b), as $\gamma$ increases, buckling amplitude decrease too. They are depending on each other.

\section{CONCLUSIONS}

The buckling and post-buckling behavior of a nanobeam with supported ends containing flowing fluid is investigated by using a nonlinear model, with the explaination of nondimensional term. An exact solution to the nonlinear equilibrium of pipes is presented. The nonlinearity of pipes is the geometric nonlinearity result from midplane stretching. A closed-form statement is concerned with for the nonlinear equilibrium configuration as a function of the fluid velocity. Clamped-hinged with torsionally spring pipe ve hybrid boundary conditions of nanobeam/nanotube is analyzed. The critical buckling velocity is obtained as a by product. The pipe is stable at its original static equilibrium position up to the flow velocity where it loses stability by static divergence by means of a supercritical pitchfork bifurcation. The straight equilibrium configuration becomes unstable and bifurcate in various equilibrium positions in the supercritical fluid velocity regime. 


\section{REFERENCES}

1. W. Yang, P. Thordarson, J. J. Gooding, S. P. Ringer,F. Braet, Carbon nanotubes for biological and biomedical applications, Nanotechnology 18, 412001-412012, 2007.

2. A. Bianco, K. Kostarelos, M. Prato, Applications of carbon nanotubes in drug delivery, Current Opinion in Chemical Biology 9, 674-679, 2005.

3. S. Adhikari, R. Chowdhury, The calibration of carbon nanotube based bionanosensors, Journal of Applied Physics 107, 124322-124329, 2010.

4. E. T. Thostenson, Z. Ren, T. W. Chou, Advances in the science and technology of carbon nanotubes and their composites: a review, Composites Science and Technology 61, 1899-1912, 2001.

5. S. Deladi, J. W. Berenschot, N. R. Tas, G. J. M. Krijnen, J. H. deBoer, M. J. deBoer, M. C. Elwenspoek Fabrication of micromachined fountain pen with in situ characterization possibility of nanoscale surface modification. Journal of Micromechanics and Microengineering 15, 528-534, 2005

6. S. Houliara, S. A. Karamanos, Buckling and post-buckling of long pressurized elastic thin-walled tubes under in-plane bending, International Journal of Non-Linear Mechanics 41(4), 491-511, 2006.

7. A. H. Nayfeh, S. A. Emam, Exact solutions and stability of the postbuckling configurations of beams, Nonlinear Dynamics 54(4), 395-408, 2008

8. H. S. Shena, C. L. Zhanga, Thermal buckling and postbuckling behavior of functionally graded carbon nanotube-reinforced composite plates, Materials \& Design 31(7), 3403-341, 2010.

9.A. R. Setoodeh, M. Khosrownejad, P. Malekzadeh, Exact nonlocal solution for postbuckling of single-walled carbon nanotubes, Physica E: Low-dimensional Systems and Nanostructures 43(9), 1730-1737, 2011.

10. L.Wang, Surface effect on buckling configuration of nanobeams containing internal flowing fluid:A nonlinear analysis, Physica E, doi:10.1016/j.physe.2011.12.006.

11. M. H. Ghayesh, M. Amabili, Post-buckling bifurcations and stability of high-speed axially moving beams, International Journal of Mechanical Sciences 68, 76-91, 2013.

12. A. Ghasemi, M. Dardel, Mohammad Hassan Ghasemi, Mohammad Mehdi Barzegari, Analytical analysis of buckling and post-buckling of fluid conveying multiwalled carbon nanotubes, Applied Mathematical Modelling 37(7), 4972-4992, 2013.

13. J. A.Wickert, Non-linear vibration of a travelling tensioned beam, International Journal of Non-Linear Mechanics 27, 503-517, 1992.

14. L. Wang, Vibration analysis of fluid-conveying nanotubes with consideration of surface effects, Physica E 43, 437-439, 2010

15. M. P. Paidoussis, G. X. Li Pipes conveying fluid: a model dynamical problem, Journal of Fluids and Structures 8, 137-204, 1993.

16. M. P. Paidoussis, Fluid-Structure Interactions: Slender Structures and Axial Flow, Vol. 1, Academic Press, London 1998. 